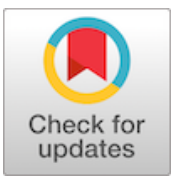

\title{
Enfermedad de Chester Erdheim costal: reporte de caso y revisión de la literatura
}

\author{
Chester Erdheim costal disease: case report and literature review
}

\author{
Ricardo Adolfo Zapata-González $\mathbb{D}$, Lennis Jazmín Bedoya-Muñoz²D, \\ Camilo Montoya-Medina ${ }^{1} \mathbb{D}$ \\ 1 Médico, especialista en Cirugía general y en Cirugía de Tórax, Clínica CardioVID, Medellín, Colombia. \\ 2 Médica general, Clínica CardioVID, Medellín, Colombia.
}

\section{Resumen}

Introducción. La enfermedad de Erdheim Chester es un trastorno infrecuente, de etiología no clara y edad de presentación variable, que se caracteriza por la proliferación anormal de histiocitos no Langerhans. Puede presentarse de forma asintomática, con manifestaciones locales o como complicaciones sistémicas. El diagnóstico se basa en las condiciones clínicas del paciente, estudios imagenológicos y el estudio anatomopatológico, mediante el cual se confirma la enfermedad.

Caso clínico. Paciente masculino con una lesión lítica en el séptimo arco costal, inicialmente asintomático y posteriormente con dolor torácico persistente. Se realizó resección y reconstrucción de la pared torácica, con resolución de la sintomatología y adecuada evolución posoperatoria. El resultado de la patología confirmó el diagnóstico de enfermedad de Erdheim Chester.

Discusión. Se presenta el caso de un paciente con compromiso costal y fractura patológica como localización y manifestación inusual de la enfermedad de Erdheim Chester, tratado quirúrgicamente de forma adecuada, y sin aparición de recidiva. Debido a la diversidad de síntomas que pueden presentary a la afectación de múltiples órganos, además de los estudios imagenológicos, la clínica debe orientar los exámenes complementarios, como electro o ecocardiograma, resonancia nuclear magnética, o angioTAC. En los pacientes asintomáticos se ha recomendado un manejo expectante y en los sintomáticos el tratamiento médico con glucocorticoides, inhibidores de BRAF e interferón alfa. El papel de la cirugía no ha sido estudiado a profundidad.

Palabras clave: Erdheim Chester; óseo; arco costal; fractura patológica; dolor torácico; tratamiento.

Fecha de recibido: 16/04/2020 - Fecha de aceptación: 24/05/2020 - Fecha de publicación en línea: 19/04/2021 Correspondencia: Lennis J. Bedoya-Muñoz, Calle 78 B \# 75-21, Clínica CardioVID, Medellín, Colombia.

Teléfono: (4) 3227090. Correo electrónico: lennisbedoya13@gmail.com

Citar como: Zapata-González RA, Bedoya-Muñoz LJ, Montoya-Medina C. Enfermedad de Chester Erdheim costal: reporte de caso y revisión de la literatura. Rev Colomb Cir. 2021;36:534-9. https://doi.org/10.30944/20117582.626

Este es un artículo de acceso abierto bajo una Licencia Creative Commons - BY-NC-ND https://creativecommons.org/licenses/by-nc$\mathrm{nd} / 4.0 /$ deed.es 


\begin{abstract}
Introduction. Erdheim Chester disease is a rare disorder of unclear etiology and variable age of presentation, characterized by abnormal proliferation of non-Langerhans histiocytes. It can present asymptomatic, with local manifestations or as systemic complications. The diagnosis is based on the clinical conditions of the patient, imaging studies, and the pathological study, through which the disease is confirmed.
\end{abstract}

Clinical case. A male patient with a lytic lesion in the seventh costal arch, initially asymptomatic and later with persistent chest pain, underwent resection and reconstruction of the chest wall, with resolution of the symptoms and adequate postoperative evolution. The pathology report confirmed the diagnosis of Erdheim Chester's disease.

Discussion. The case of a patient with rib involvement and pathological fracture is presented as an unusual location and manifestation of Erdheim Chester's disease, treated surgically appropriately, and without recurrence. Due to the diversity of symptoms that they can present and the involvement of multiple organs, in addition to imaging studies, the clinic should direct complementary tests such as electrocardiogram or echocardiogram, magnetic resonance imaging, or CT angiography. Expectant management has been recommended in asymptomatic patients, and medical treatment with glucocorticoids, BRAF inhibitors, and interferon alfa in symptomatic patients. The role of surgery has not been studied in depth.

Keywords: Erdheim Chester; osseous; costal arch; pathological fracture; chest pain; treatment.

\section{Introducción}

La enfermedad de Erdheim Chester, una histiocitosis de células no Langerhans, es un trastorno poco común, que afecta principalmente a hombres entre la quinta y la séptima década de la vida ${ }^{1}$. El primer caso descrito fue en 1930 por Jakob Erdheim y William Chester. Hasta el 2014 se habían reportado en la literatura aproximadamente 500 casos $^{2}$.

Su fisiopatología es poco clara, no se han encontrado marcadores genéticos que predispongan a la enfermedad, ni procesos infecciosos que lo generen, sin embargo, el desarrollo de la enfermedad se ha relacionado con la proliferación exagerada de las células $\mathrm{T}^{2}$. Se ha incluido en un grupo raro de neoplasias mieloides inflamatorias, las cuales se caracterizan por mutaciones en la vía de señalización regulada por las quinasas, y que involucra el gen $\mathrm{BRAF}^{3}$.

Las manifestaciones clínicas de este trastorno son heterogéneas, desde pacientes asintomáticos, hasta la presentación de compromiso multiorgánico ${ }^{1}$. La afección ósea es la principal manifestación clínica, presente hasta en el $75 \%$ de los pacientes, que generalmente se localiza a nivel de las diáfisis de huesos largos, de forma simétrica y bilateral. Puede tener manifestaciones extraóseas como exoftalmos, diabetes insípida, enfermedad pulmonar intersticial, compromiso adrenal bilateral, fibrosis retroperitoneal, insuficiencia renal, infiltración testicular y compromiso del sistema nervioso central y cardiovascular ${ }^{4}$. Por sus múltiples formas de presentación el diagnóstico es un reto, sin embargo, la combinación de manifestaciones clínicas, los hallazgos imagenológicos y la histopatología permiten confirmar la enfermedad.

De acuerdo con el compromiso del paciente se debe seleccionar la imagen que ayude a estrechar el espectro diagnóstico diferencial. La radiografía de tórax y la gammagrafía ósea son útiles cuando existe compromiso óseo de las extremidades, mientras que la tomografía y la resonancia magnética son los exámenes de elección en la región toracoabdominal. La ecografía o imágenes nucleares pueden ser exámenes complementarios ${ }^{5}$. La tomografía por emisión de positrones (PET-TC, por sus siglas en inglés) es útil tanto para el diagnóstico como para el seguimiento, y permite además determinar la extensión de la enfermedad ${ }^{6}$. La histopatología es el examen confirmatorio del diagnóstico y en ella se observan infiltrados histiocitarios granulomatosos $\mathrm{y}$ polimórficos o fibrosis xantogranulomatosa con 
tinciones de inmunohistoquímica CD68-positiva y CD1a-negativa ${ }^{2,7,8}$.

El objetivo de este artículo fue presentar el caso de un paciente de 59 años con una lesión lítica en el séptimo arco costal, en quien se realizó resección quirúrgica con reconstrucción de la pared, confirmándose histológicamente el diagnóstico de la enfermedad de Erdheim Chester. Se realiza una revisión de la literatura.

\section{Caso clínico}

Paciente masculino de 59 años de edad, con antecedente de hipertensión arterial, hipertrigliceridemia y aterosclerosis calcificada con prueba de perfusión miocárdica negativa para isquemia. Fue valorado en la clínica CardioVID, en Medellín, Colombia, por dolor torácico izquierdo de tres meses de evolución, se realizó una tomografía de tórax, observando una lesión lítica de borde escleroso de 13 x $6 \mathrm{~mm}$, localizada en la cara lateral del séptimo arco costal izquierdo, con engrosamiento del espacio pleural y ruptura de la cortical medial y lateral, sin masa sólida acompañante (figura 1). La biopsia por punción guiada por tomografía fue negativa para malignidad y se descartaron lesiones metastásicas, por lo que inicialmente se decidió realizar seguimiento tomográfico anual.

Posteriormente el paciente presentó aumento progresivo del dolor torácico, asociado a limitación funcional, sin cambios imagenológicos de la masa, pero con aparición de una fractura patológi- ca asociada a la lesión, por lo que se llevó a cirugía. Mediante una incisión en piel sobre el séptimo arco costal, se identificó una lesión de aproximadamente $1,5 \mathrm{~cm}$, que abombaba la cortical, sin infiltración de los tejidos adyacentes. Se resecó la lesión y el séptimo arco costal, preservando un margen anterior y posterior de $3 \mathrm{~cm}$, para posteriormente reconstruir la pared torácica con una barra de titanio y una malla de polipropileno, para evitar el riesgo de herniación pulmonar. Se verificó la adecuada expansión pulmonar y se realizó el cierre muscular por planos. No hubo complicaciones y el posoperatorio transcurrió de forma adecuada. Las radiografías de tórax de control no mostraron hallazgos patológicos ni recidiva de la enfermedad (figura 2).

El resultado de patología informó una lesión intramedular bien delimitada, constituida por abundantes histiocitos vacuolados con núcleos redondos y algunas células multinucleadas gigantes, rodeado de escasos cristales de colesterol con focos de fibrosis estromal circundantes y signos de reabsorción y remodelación ósea, además de un discreto infiltrado mononuclear. Estos cambios correspondieron a un desorden xantogranulomatoso infiltrativo compatible con la enfermedad de Erdheim Chester. Las pruebas de inmunohistoquímica CD68 positiva, CD163, CD1A y CK negativas, confirmaron el diagnóstico. Después de 2 años de seguimiento el paciente está asintomático y los controles radiológicos son normales (Figura 3).

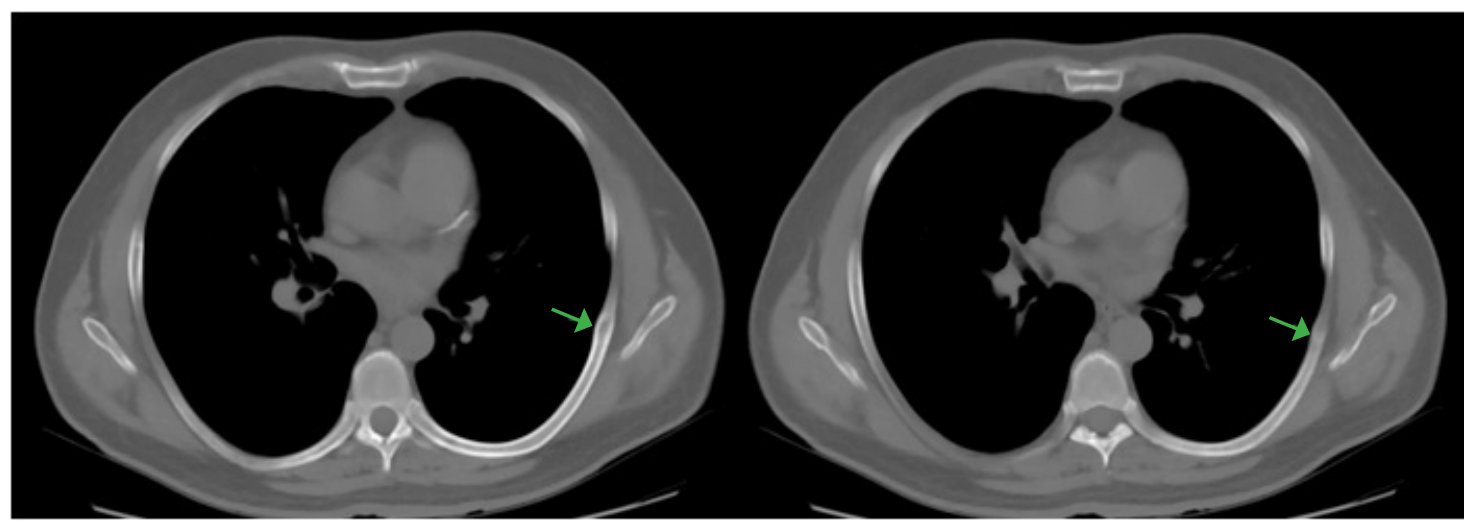

Figura 1. Corte axial de la tomografía en la que se observa la lesión lítica en el séptimo arco costal (flecha verde). 


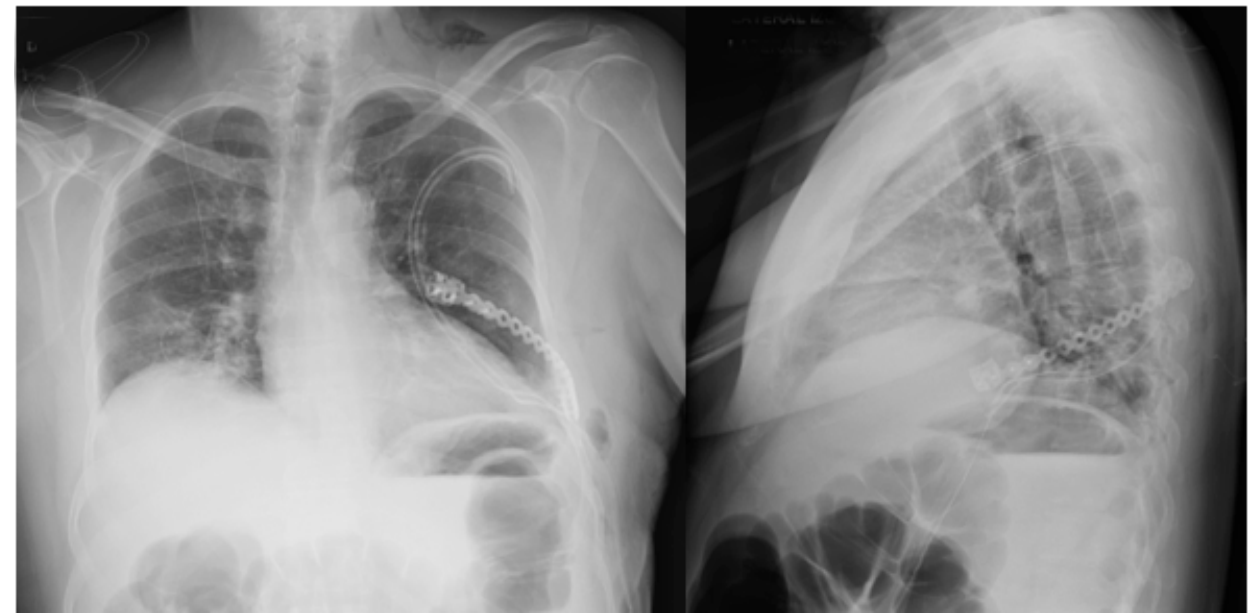

Figura 2. Radiografía anteroposterior y lateral de tórax al primer día postoperatorio

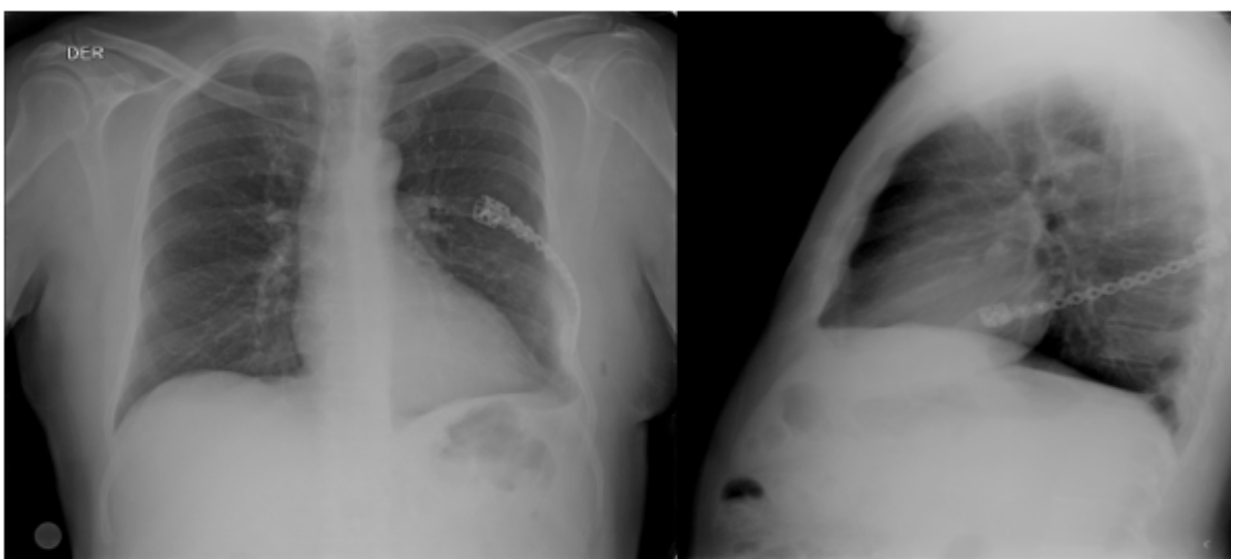

Figura 3. Radiografía de tórax normal al segundo año de control posoperatorio.

\section{Discusión}

La enfermedad de Erdheim Chester es una patología poco común, que corresponde a menos del $1 \%$ de las neoplasias de tejidos blandos y ganglios linfáticos ${ }^{7}$. Se caracteriza por la acumulación de macrófagos espumosos, inflamación crónica y fibrosis. En 2016 la Organización Mundial de la Salud la clasificó como una neoplasia hematopoyética de origen histiocito de células no Langerhans ${ }^{9}$.

Tiene una baja prevalencia, pero su incidencia ha aumentado en los últimos años, probablemente por la mayor disponibilidad de recursos y conocimiento de la enfermedad. Su presentación es un poco mayor en hombres que en mujeres, con una relación 3 a 1, y es más frecuente en la sexta década de la vida, lo cual coincide con el sexo y la edad del paciente comentado. En la literatura se han reportado algunos casos en pacientes pediátricos, pero la enfermedad es menos frecuente en los extremos de la vida ${ }^{10}$.

La presentación clínica es inespecífica e incluso puede cursar de forma asintomática, esto hace que su diagnóstico sea difícil y frecuentemente tardío. En cuanto a las ayudas diagnósticas, el espectro imagenológico es amplio, y el tipo de examen solicitado debe estar orientado de acuerdo 
con el compromiso del paciente. Las alteraciones esqueléticas ocurren en el $96 \%$ de los pacientes, por lo tanto, se deben evaluar siempre que se sospeche la enfermedad. En la mayoría de los casos el compromiso óseo ocurre en las extremidades, principalmente en las diáfisis de los huesos largos de los miembros inferiores ${ }^{5}$, pero también puede comprometer otras estructuras como en el caso de este paciente.

En la radiografía de tórax se puede observar esclerosis de la cortical como un signo inespecífico. En el paciente presentado se encontró además una fractura patológica, hallazgo inusual, que debe ser considerado un signo de alarma, e implica la realización de estudios adicionales para buscar exhaustivamente el diagnóstico. La tomografía se usa ampliamente, tanto para el diagnóstico como para el seguimiento de los pacientes, dado que permite una mejor caracterización de la lesión y la toma de conductas adicionales ${ }^{11}$.

El PET-TC ha sido recomendado para el estudio de extensión de la enfermedad, para detectar el compromiso visceral y vascular, la actividad de la lesión y el compromiso a distancia, dado que permite observar otros sitios que presenten actividad metabólica aumentada ${ }^{9-12}$. Este examen debe realizarse en pacientes con síntomas no localizados ${ }^{13}$. En el caso mencionado, el paciente no presentaba signos o síntomas de compromiso a distancia, por lo que no fue necesario realizar estudios de extensión. Se ha realizado un seguimiento estricto del paciente y persiste asintomático.

Debido a la diversidad de síntomas que pueden presentar los pacientes y a la afectación de múltiples órganos, la clínica debe orientar otros exámenes complementarios, como electrocardiograma y ecocardiografía, si presenta síntomas cardiacos; resonancia nuclear magnética (RNM) cerebral, ante la sospecha de compromiso del sistema nervioso central; RNM o angioTAC, si se sospecha aortitis o compromiso vascular ${ }^{14}$. El paciente previamente descrito no presentaba este tipo de síntomas por lo que no requirió estudios complementarios, solo se realizó un ecocardiograma debido al dolor torácico inicial y como estudio prequirúrgico, que fue completamente normal.
La literatura recomienda el tratamiento médico con glucocorticoides, inhibidores de BRAF e interferón alfa en pacientes sintomáticos, con compromiso del sistema nervioso central y con disfunción orgánica. En los pacientes asintomáticos los reportes de caso y los estudios han recomendado un manejo expectante. El papel de la cirugía no ha sido evaluado a profundidad para pacientes con enfermedad de Erdheim Chester, reservándola únicamente en casos donde se observa presencia de complicaciones, como daño valvular cardiaco ${ }^{15,16}$. En nuestro caso se trató de un paciente sintomático, con fractura patológica a nivel costal, en quien el manejo quirúrgico logró, no solo la mejoría completa de los síntomas, sino la curación. Durante el seguimiento del paciente no se ha evidenciado recidiva de la enfermedad.

\section{Conclusión}

La enfermedad de Erdheim Chester es una patología poco frecuente. Se presenta el caso de un paciente con compromiso costal, localización inusual de esta enfermedad, asociada a fractura patológica, otro hallazgo poco común en este tipo de anomalías.

El tratamiento quirúrgico con resección amplia de la lesión y reconstrucción con material de titanio fue curativo, logrando remisión completa de los síntomas y sin recidiva de la enfermedad tras dos años de seguimiento.

\section{Cumplimiento de normas éticas}

Consentimiento informado: Este estudio es la presentación de un caso con una revisión de historia clínica retrospectiva, y como tal, no hay necesidad de un consentimiento informado.

Declaración de conflicto de intereses: Los autores declaran que no tienen conflicto de intereses.

Fuentes de financiación: Recursos propios de los autores.

\section{Declaración de contribución de los autores}

- Concepción y diseño del estudio: Ricardo Adolfo ZapataGonzález.

- Adquisición de datos: Ricardo Adolfo Zapata-González, Lennis Jazmín Bedoya-Muñoz y Camilo Montoya-Medina. 
- Análisis e interpretación de datos: Ricardo Adolfo Zapata-González, Lennis Jazmín Bedoya-Muñoz y Camilo Montoya-Medina.

- Redacción del manuscrito: Ricardo Adolfo ZapataGonzález, Lennis Jazmín Bedoya-Muñoz y Camilo Montoya-Medina.

- Revisión crítica: Ricardo Adolfo Zapata-González y Camilo Montoya-Medina.

\section{Referencias}

1. Cives M, Simone V, Rizzo FM, Dicuonzo F, Cristallo Lacalamita M, Ingravallo G, et al. Erdheim-Chester disease: a systematic review. Crit Rev Oncol Hematol. 2015;95:111. https://doi.org/10.1016/j.critrevonc.2015.02.004

2. Haroche J, Arnaud L, Cohen-Aubart F, Hervier B, Charlotte F, Emile JF, et al. Erdheim-Chester disease. Curr Rheumatol Rep. 2014;16:412. https://doi.org/10.1007/s11926-014-0412-0

3. Milne P, Bigley V, Bacon CM, Néel A, McGovern N, Bomken $\mathrm{S}$, et al. Hematopoietic origin of Langerhans cell histiocytosis and Erdheim-Chester disease in adults. Blood. 2017;130:167-175. https://doi.org/10.1182/blood-2016-12-757823

4. Nakhleh A, Slobodin G, Elias N, Bejar J, Odeh M. Rapid progression to cardiac tamponade in Erdheim-Chester disease despite treatment with interferon alpha. Mod Rheumatol. 2016;26:621-4. https://doi.org/10.3109/14397595.2014.905235

5. Kumar P, Singh A, Gamanagatti S, Kumar S, Chandrashekhara SH. Imaging findings in Erdheim-Chester disease: what every radiologist needs to know. Pol J Radiol. 2018;83:e54-e62. https://doi.org/10.5114/pjr.2018.73290

6. Sabino D, do Vale RHB, Duarte PS, Sapienza MT, Buchpiguel CA. Complementary findings on (18)F-FDG PET/CT and (18)F-NaF PET/CT in a patient with Erdheim-Chester disease. Radiol Bras. 2017;50:202-3. https://doi.org/10.1590/0100-3984.2015.0172

7. Durham BH. Molecular characterization of the histiocytoses: neoplasia of dendritic cells and macrophages. Semin Cell Dev Biol. 2018;86:62-76. https://doi.org/10.1016/j.semcdb.2018.03.002

8. Ozkaya N, Rosenblum MK, Durham BH, Pichardo JD, Abdel-Wahab 0, Hameed MR, et al. The histopathology of Erdheim-Chester disease: a comprehensive review of a molecularly characterized cohort. Moder Pathol. 2017;31:581-97. https://doi.org/10.1038/modpathol.2017.160

9. Estrada-Veras JI, O’Brien KJ, Boyd LC, Dave RH, Durham B, Xi L, et al. The clinical spectrum of Erdheim-Chester disease: an observational cohort study. Blood Adv. 2017;1:357-66.

https://doi.org/10.1182/bloodadvances.2016001784

10. Haroche J, Cohen-Aubart F, Rollins BJ, Donadieu J, Charlotte F, Idbaih A, et al. Histiocytoses: emerging neoplasia behind inflammation. Lancet Oncol. 2017;18:e113-e125. https://doi.org/10.1016/S1470-2045(17)30031-1

11. Arnaud L, Malek Z, Archambaud F, Kas A, Toledano D, Drier A, et al. 18F-fluorodeoxyglucose-positron emission tomography scanning is more useful in follow up than in the initial assessment of patients with Erdheim-Chester disease. Arthritis Rheum. 2009;60:312838. https://doi.org/10.1002/art.24848

12. Steňová E, Steňo $B$, Povinec $P$, Ondriaš F, Rampalová J. FDG-PET in theErdheim-Chester disease: its diagnostic and follow-up role. Rheumatol Int. 2012;32:675-8. https://doi.org/10.1007/s00296-010-1676-y

13. Anelli M, Smith M, Mengoli MC, Spaggiari L, De Marco L, Lococo F, et al. Erdheim Chester Disease: description of 2 illustrative cases involving the lung. Histopathology. 2018;73:167-72. https://doi.org/10.1111/his.13501

14. Diamond EL, Dagna L, Hyman DM, Cavalli G, Janku F, Estrada-Veras J, et al. Consensus guidelines for the diagnosis and clinical management of Erdheim-Chester disease. Blood. 2014;124:483-92. https://doi.org/10.1182/blood-2014-03-561381

15. Arnaud L, Hervier B, Néel A, Hamidou M, Kahn JE, Wechsler B, et al. CNS involvement and treatment with interferon- $\alpha$ are independent prognostic factors in Erdheim-Chester disease: a multicenter survival analysis of 53 patients. Blood 2011;117:2778-82. https://doi.org/10.1182/blood-2010-06-294108

16. Haroche J, Cohen-Aubart F, Emile JF, Arnaud L, Maksud $\mathrm{P}$, Charlotte F, et al. Dramatic efficacy of vemurafenib in both multisystemic and refractory Erdheim-Chester disease and Langerhans cell histiocytosis harboring the BRAF V600E mutation. Blood. 2013;121:1495-1500. https://doi.org/10.1182/blood-2012-07-446286 